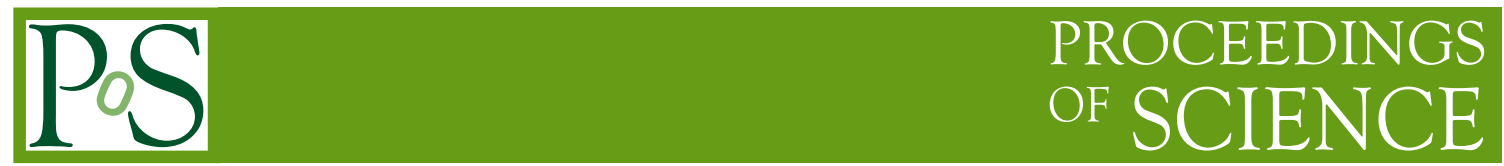

\title{
Constructing the liquid-vapor phase diagram of infinite nuclear matter
}

\section{Larry Phair*, P. T. Lake, and L. G. Moretto}

Nuclear Science Division, Lawrence Berkeley National Laboratory,

Berkeley, CA, USA

E-mail: lwphairelbl.gov

\section{J. B. Elliott}

Lawrence Livermore National Laboratory

Livermore, CA, USA

\begin{abstract}
We have made an estimate of the critical properties of infinite uncharged nuclear matter based on experimental data. To do this, three obstacles had to be overcome: finite size effects, the Coulomb interaction, and an appropriate physical picture (not particles coexisting in a box) that reflects particle emission into a vacuum.
\end{abstract}

8th International Workshop on Critical Point and Onset of Deconfinement March 11-15

Napa, California, USA

\footnotetext{
* Speaker.
} 


\section{Introduction}

After decades of theoretical and experimental studies, recent papers have published what can be considered a quantitative, credible liquid-vapor phase diagram of nuclear matter containing the coexistence line up to the critical temperature [1,2]. Somewhat unexpectedly, this diagram has not been obtained through the study of caloric curves [3,4] or anomalous heat capacities [5,6], but rather through the fitting of the charge distributions in multifragmentation by means of a Coulombcorrected Fisher's formula [1, 7] giving the cluster composition of a vapor:

$$
n_{A}(T)=q_{0} A^{-\tau} \exp \left[\frac{\Delta \mu A}{T}-\frac{c_{0} \varepsilon A^{\sigma}}{T}\right],
$$

where $q_{0}$ is a normalization constant [7]; $\tau$ is the critical exponent giving rise to a power law at criticality; $A$ is the cluster number; $\Delta \mu$ is the difference of chemical potentials between the liquid and the vapor; $c_{0}$ is the surface energy coefficient; $T$ is the temperature; $\varepsilon=\left(T_{c}-T\right) / T_{c}$ is the distance from the critical temperature $T_{c} ; \sigma$ is another critical exponent (expected to be approximately 2/3). For $\Delta \mu=0$ the liquid and the vapor are in equilibrium and Eq. (1.1) can be taken to be the equivalent of the cluster concentrations at coexistence. More conventionally, one can immediately obtain from Eq. (1.1) the usual $p, T$ and $\rho, T$ phase diagrams by recalling that in Fisher's model, the clusterization is assumed to exhaust all the non-idealities of the gas. It then becomes an ideal gas of clusters. Consequently, the total pressure and density are

$$
\begin{gathered}
p(T)=\sum_{A} p_{A}(T)=T \sum_{A} n_{A}(T), \\
\rho=\sum_{A} A n_{A}(T),
\end{gathered}
$$

as well as the corresponding scaled quantities $p / p_{c}$ and $\rho / \rho_{c}$.

In order to make use of the Fisher description of the cluster concentrations there were three major obstacles to overcome: finite size effects, the problem of the long-range Coulomb interaction, and the (un)physical picture of particles in a box.

\section{Finite size effects}

In the nuclear case finite size effects dominate the picture macroscopically and spectroscopically at all excitation energies. The challenge is to reduce the vast amount of specific knowledge of each "drop" (nucleus) to a general characterization of uncharged, symmetric infinite nuclear matter. This reduction has been successfully achieved for cold nuclei $(T=0)$ in determining the saturation binding energy and density by means of the liquid drop model.

We have developed a general method to deal with finite size effects in phase transitions [8]. In the case of liquid-vapor phase coexistence, a dilute nearly ideal vapor phase is in equilibrium with a dense liquid-like phase. The interesting case of finiteness is realized when the liquid phase is a finite drop. The vapor pressure of a drop can be calculated by correcting the molar vaporization enthalpy to include the surface energy of the drop [9]. We introduced the concept of the complement (the residual drop which remains after a cluster has been emitted) in order to further quantify finite size effects and to generalize Fisher's theory to better describe the cluster yields from extremely small 
systems [8]. The complement approach consists of evaluating the change in free energy occuring when a particle or cluster is moved from one (finite) phase to another. In the case of a liquid drop in equilibrium with its vapor, this is done by extracting a vapor particle of any given size from the drop and evaluating the energy and entropy changes associated with both the vapor particle and the residual liquid drop (the complement).

This detailed accounting can be easily generalized to incorporate other energy terms common in the nuclear case, such as symmetry energy, Coulomb energy and even angular momentum [1, $2,10]$. In order to demonstrate the power of this method, we apply it to the Ising model, where a great deal of work exists on the subject of finiteness, in particular on the dependence of critical quantities on the lattice size [11, 12].

In the canonical lattice gas representation of the Ising model, a fixed number of up spins represents the occupied lattice sites (i.e., matter in the form of monomers, dimers, large drops etc.), while down spins are empty space. In a finite lattice, we can fix the mean density of occupied sites in such a way that, below the coexistence temperature $T_{0}$, there is a large cluster or drop of a certain size in equilibrium with its vapor, populated mostly by monomers. Two dimensional Ising calculations were performed for fixed magnetization (equivalently, fixed mean occupation density). Periodic boundary conditions were used to minimize the finite size effects of the container. At the lowest temperatures the up spins congregate into one liquid drop in a vacuum. At higher temperatures, the vacuum is filled with a vapor made from clusters of up spins. Clusters in the vapor were identified via the Coniglio-Klein algorithm [13] to insure that they were physical (i.e., cluster distributions return Ising critical exponents and not percolation exponents). The largest cluster was formed via a geometric clustering algorithm (all spin up nearest neighbors are bonded together) and identified as the liquid drop. The largest cluster is identified geometrically and not via the Coniglio-Klein prescription because we do not want to include clusters associated with the skin thickness of the liquid into our description of the vapor [14].

Rather than consider the vapor globally, we examine the vapor in detail. Fisher's droplet model [7] describes the cluster concentration and can be written as

$$
\begin{aligned}
n_{A}(T) & =g(A) \exp \left(-\frac{c_{0} A^{\sigma}}{T}\right) \\
& =q_{0} A^{-\tau} \exp \left(c_{0} A^{\sigma}\left[\frac{1}{T_{c}}-\frac{1}{T}\right]\right)
\end{aligned}
$$

where $q_{0}$ is the normalization, $c_{0} A^{\sigma}$ is the surface energy of the cluster and $g(A)$ is the degeneracy of the clusters of size $A$ (the number of ways the cluster $A$ can be realized through different surface configurations) and is approximately $A^{-\tau} \exp \left(c_{0} A^{\sigma} / T_{c}\right)$. The resulting surface entropy $S(A)$ is given by

$$
S(A) \approx \ln g(A) \approx-\tau \ln A+\frac{c_{0} A^{\sigma}}{T_{c}}
$$

Eq. (2.2) is a remarkably felicitous asymptotic expansion. The presence of a leading term in $S$ proportional to $A^{\sigma}$ permits the vanishing of the cluster free energy at a $T=T_{c}$ independent of cluster size. This expression, valid for a vapor in equilibrium with the infinite liquid, must be generalized for equilibrium with a finite liquid. This can be done by making a preliminary observation: in Fisher's expression the abundance of each cluster in the vapor follows a Poisson distribution by 
construction. This is because the resulting partial vapors are considered as non-interacting. In the Ising/lattice gas model this same situation prevails to a surprising degree (as can be seen in ref. [15]). The Poisson nature of the multiplicity distributions allows us to introduce the concept of the complement.

Consider a vapor in equilibrium with a drop of its liquid. The system may be a physical system or the Ising realization of it. For each cluster of the vapor we can make the mental exercise of extracting it from the liquid, determining the change in entropy and energy of the drop and cluster system, and then putting it back in the liquid (the equilibrium condition), as if all other clusters of the vapor did not exist. Fisher's expression can now be written for a drop of size $A_{0}$ in equilibrium with its vapor as follows

$$
\begin{aligned}
n_{A}(T)= & \frac{g(A) g\left(A_{0}-A\right)}{g\left(A_{0}\right)} \\
& \exp \left\{-\frac{c_{0}}{T}\left[A^{\sigma}+\left(A_{0}-A\right)^{\sigma}-A_{0}^{\sigma}\right]\right\}
\end{aligned}
$$

or

$$
\begin{aligned}
n_{A}(T)= & q_{0}\left[\frac{A\left(A_{0}-A\right)}{A_{0}}\right]^{-\tau} \\
& \exp \left\{\left[A^{\sigma}+\left(A_{0}-A\right)^{\sigma}-A_{0}^{\sigma}\right]\left(\frac{c_{0}}{T_{c}}-\frac{c_{0}}{T}\right)\right\} .
\end{aligned}
$$

In other words, we treat the "complement" $\left(A_{0}-A\right)$ in the same fashion as a cluster. The resulting expression reduces to Eq. (2.1) when $A_{0}$ tends to infinity. While different than the standard Fisher expression, Eq. (2.4) still admits the same $T_{c}$ as that of the infinite system. This is because the $A_{0}$, $A$ dependence of the surface energy finds its exact counterpart in that of the surface entropy.

We are now prepared to compare the Ising yields of the vapor concentrations to our modified Fisher's droplet model. An example is given in Fig. 1. The yields in the upper panel show the linear Boltzmann behavior associated with a first order phase transition. We do not show temperatures above $T=1.65$ because for those temperatures there is no coexisting liquid drop in the calculations - it has vaporized. Fisher's description is intended to describe the coexistence condition and is shown by the solid lines of Fig. 1. In the lower panel is shown the scaling condition where the ordinate is scaled by the Fisher power law and the abscissa is scaled to reflect the total surface energy over the temperature. The scaling works rather well.

From the Fisher droplet model fits to the yields comes an estimate of the critical temperature of $2.32 \pm 0.02$. This is to be compared to the known analytical solution of $T_{c}=2 / \ln (1+\sqrt{2})=$ 2.269 ... Without the complement correction to the Fisher droplet model, a fit gives results of similar quality but a critical temperature of $2.07 \pm 0.05$, clearly in worse agreement with the known theoretical value.

The line represented by the scaled behavior shown in the lower panel of Fig. 1 contains all the information regarding coexistence between the finite liquid and vapor phases in the lattice gas model. Using that information, the coexistence pressure and density can be calculated (see the solid line in Fig. 2). The agreement between the Fisher description and the Ising calculation (open symbols) is quite good. Furthermore, one can take the finite value for $A_{0}$ used in the modified Fisher droplet model of Eq. (2.4), and let it go to infinity to recover the standard Fisher description 

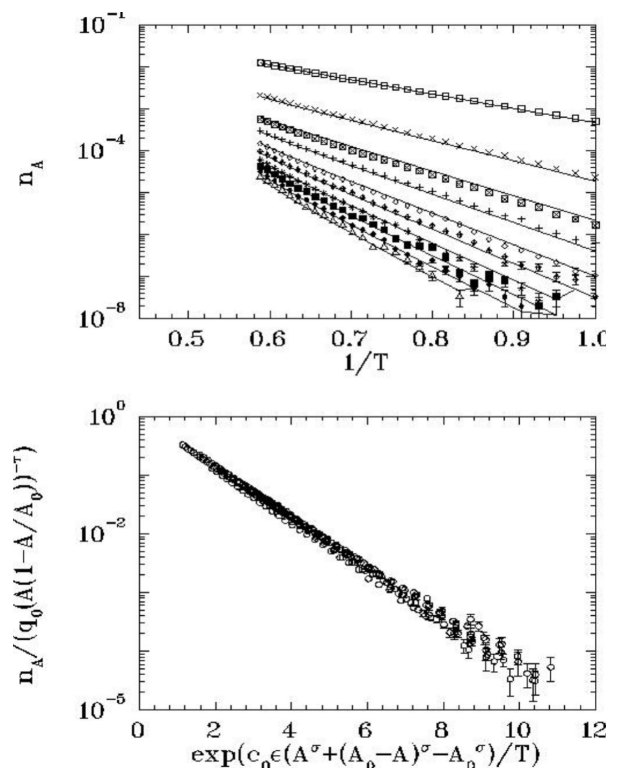

Figure 1: Upper panel: the yields of clusters of size one (open squares) up to clusters of size ten (open triangles) as a function of temperature from a two dimensional lattice gas calculation (lattice of 40 by 40 ) at constant density $\rho=0.2$ (corresponding to a cold liquid drop of $A_{0}=80$ ) as a function of $1 / T$. The lines are fits to the yields using the modified Fisher droplet model described in Eq. (2.4). Lower panel: the same yields scaled by the power law on the ordinate and the surface energy over temperature on the abscissa.

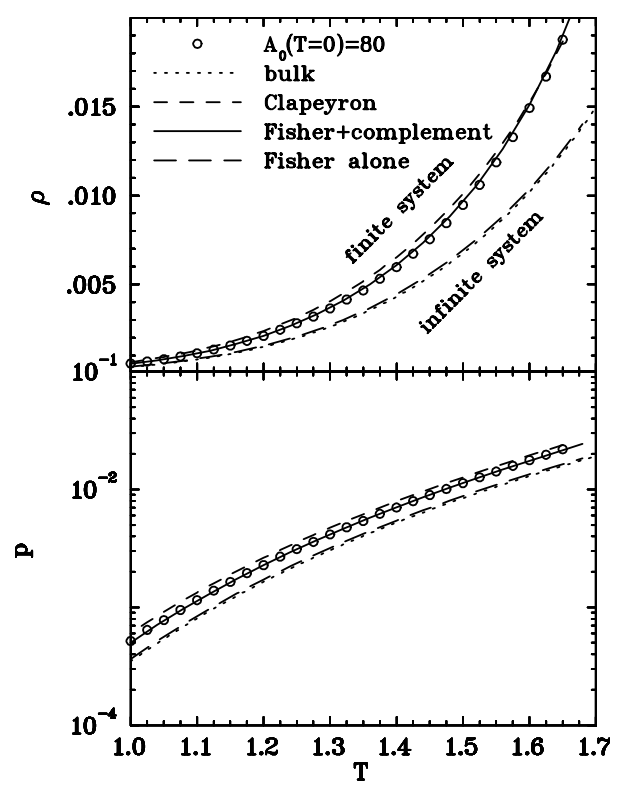

Figure 2: The density $\rho$ and pressure $p$ of a vapor in coexistence with a finite drop $A_{0}$ whose size at $T=0$ is 80 (symbols) from $2 \mathrm{~d}$ Ising calculations at fixed magnetization. The dotted lines are the analytic bulk solutions known for the Ising model in two dimensions [16]. The solid line comes from fits to the cluster yields using the modified Fisher formula to include the complement (see Eq. (2.4)). The long dashed curve comes from the normal Fisher description (see Eq. (2.1)) of the cluster yields. 
for coexistence with an infinite liquid. This is shown by the long dashed curve in Fig. 2. The agreement with the known analytic solution of Onsager [16] (dotted curve) is remarkable. We have a practical way of now moving from finite to infinite systems with the Fisher droplet model.

\section{Coulomb}

In ref. [10], we explored the effects of the Coulomb interaction upon the nuclear liquid phase transition. Because large nuclei are metastable objects, phases, phase coexistence, and phase transitions cannot be defined with any generality and the analogy to liquid vapor is ill-posed for these heavy systems. However, it is possible to account for the Coulomb interaction in the decay rates and obtain the coexistence phase diagram for the corresponding uncharged system [1].

\section{Physical picture}

We now specifically address fragment emission from an excited nucleus with a time-honored assumption which we do not justify other than through the clarification it brings to the experimental picture: we assume (just as in compound nuclear decay) that after prompt emission in the initial phase of the collision, the resulting system relaxes in shape and density and thermalizes on a time scale shorter than its thermal decay. At this point, the excited nucleus emits particles in vacuum, according to standard statistical decay rate theory. In this picture, there is no surrounding vapor and no confining box; there is no need for either. By studying the outward flux of the first fragments emitted, we can study the nature of the vapor even when it is absent (the virtual vapor) because of the equivalence of the evaporation and condensation fluxes of a liquid in equilibrium with its saturated vapor. Quantitatively, the concentration $n_{A}(T)$ of any species $A$ in the vapor is related to the corresponding decay rate $R_{A}(T)$ (or to the decay width $\Gamma_{A}$ ) from the nucleus by matching the evaporation and condensation fluxes

$$
R_{A}(T)=\frac{\Gamma_{A}(T)}{\hbar} \approx n_{A}(T)\left\langle v_{A}(T) 4 \sigma_{\mathrm{inv}}\left(v_{A}\right)\right\rangle,
$$

where $v_{A}(T)$ is the velocity of the species $A$ (of order $(T / A)^{1 / 2}$ ) crossing the nuclear interface represented by the inverse cross section $\sigma_{\text {inv }}$. The temperature $T_{0}$ of the equilibrated, excited nucleus when the first fragment is emitted can be estimated by the thermometric equation of a Fermi gas and the calorimetrically measured excitation energy $E^{*}$ such that $T_{0}=\sqrt{E^{*} / a}$, allowing for a weak dependence of $a$ on $T$ and remembering that the system is most likely still in the Fermi strong degeneracy regime (where the temperature is much less than the Fermi energy: $T<<\varepsilon_{F}$ ).

This is the fundamental and simple connection between Eq. (4.1), the (compound nucleus) decay rate, and Eq. (2.4), the modified Fisher droplet model. In the latter, one immediately recognizes in the exponential the canonical expansion of the standard compound nucleus decay rate, namely, the Boltzmann factor. The surface part of the barrier is isolated from all other components, e.g., Coulomb [10], symmetry, and finite size [8]. Thus, the vapor phase in equilibrium can be completely characterized in terms of the decay rate.

The physical picture described above is valid instantaneously, but not globally. The result of a global or successive evaporation in vacuum leads to abundances of various species of emitted 


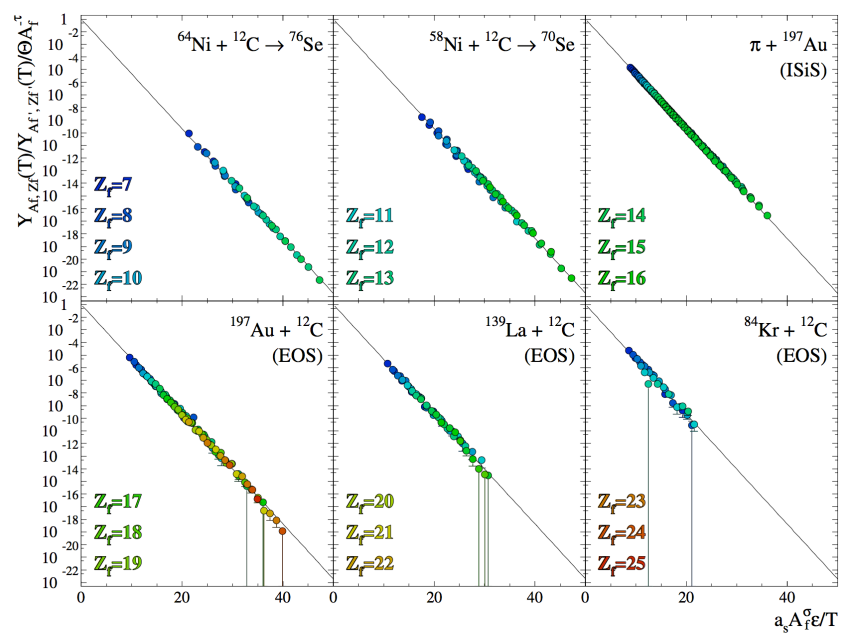

Figure 3: The scaled charge yields for the six indicated reactions. Over 500 points are collapsed onto a single curve which describes the behavior of bulk nuclear matter. The color of the points shows the charge of the fragments. The solid line shows the liquid-vapor coexistence curve of bulk nuclear matter. Here $\Theta$ is an effective chemical potential that depends on the effects of finite size, the Coulomb force, angular momentum and isospin. See [1] for further details.

fragments that arise from a continuum of systems at different temperatures [17]. This leads to complications in various thermometers: kinetic energy, isotope ratios, etc. One way to avoid this complication is to consider only fragments that are emitted very rarely so that, if they are not emitted first, they are effectively not emitted at all. In other words, we consider only fragments that by virtue of their large surface energy have a high emission barrier.

\section{Infinite nuclear matter}

With solutions for the problems posed by finite size effects and the long-range Coulomb interaction along with an appropriate physical picture, we are ready to attempt a Fisher description of yields measured in different nuclear reactions.

Having developed and tested in so far as possible this systematic approach, we have applied it to a series of nuclear reactions: low energy compound nucleus decay and higher energy multifragmentation. Low energy compound nuclear reactions were particularly important because the mass, charge, excitation energy and angular momentum of the decaying system could easily be determined.

Results are shown in Fig. 3 for the indicated reactions. When the yields are scaled by the Coulomb, rotational, symmetry energies, etc. (all terms that are not surface energy), and plotted as a function of the Boltzmann factor associated with surface energy, the different reactions show a similar scaling. This scaled line contains all the information of coexistence between the two phases for chargeless, symmetric, and infinite nuclear matter. The data can be fit to obtain an estimate of $T_{c}$ and such a procedure yields $T_{c}=17.9 \pm 0.4 \mathrm{MeV}$. Using the ideal gas approximations (Eqs. (1.2) and (1.3)), estimates can be obtained for the critical density $\rho_{c}$ and critical pressure $p_{c}$. Such calculations give $\rho_{c}=0.06 \pm 0.02 \mathrm{fm}^{-3}$ and $p_{c}=0.31 \pm 0.07 \mathrm{MeV} / \mathrm{fm}^{3}$. 
With the results described herein and with the more detailed analysis described in [1, 2], we have made a definitive, experimentally based measure of the phase diagram of infinite, neutral nuclear matter. Future work will explore the sensitivity of these results to model assumptions.

\section{References}

[1] J. B. Elliott et al., Phys. Rev. C 87, 054622 (2013).

[2] L. G. Moretto et al., Journal of Phys. G - Nuc. and Part. Phys. 38, 11 (2011).

[3] J. Pochodzalla et al., Phys. Rev. Lett. 75 , 1040 (1995).

[4] Chernomoretz et al., Phys. Rev. C 64, 044605 (2001).

[5] F. Gulminelli et al., Phys. Rev. Lett. 82, 1402 (1999).

[6] M. Dagostino et al., Phys. Lett B 473, 219 (2000).

[7] M. E. Fisher, Rep. Prog. Phys. 30, 615 (1969).

[8] L. G. Moretto et al., Phys. Rev. Lett. 94, 202701 (2005).

[9] L. Rayleigh, Philos. Mag. 34, 94 (1917).

[10] L. G. Moretto et al., Phys. Rev. C 68, 061602 (2003).

[11] M. E. Fisher and A. E. Ferdinand, Phys. Rev. Lett. 19, 169 (1967).

[12] D. P. Landau, Phys. Rev. B 13, 2997 (1976).

[13] A. Coniglio and W. Klein, J. Phys. A 13, 2775 (1980).

[14] J. B. Elliott et al., Phys. Rev. C 71, 024607 (2005).

[15] C. M. Mader et al., Phys. Rev. C 68, 064601 (2003).

[16] L. Onsager, Phys. Rev. 65, 117 (1944).

[17] L. G. Moretto et al., Phys. Rev. C 72, 064605 (2005). 